\title{
Curso de Enfermagem da Universidade Federal de luiz de Fora: 1977-1979
}

\author{
The Nursing Course of Universidade Federal de Juiz de Fora: 1977-1979
}

Curso de Enfermería de la Universidade Federal de Juiz de Fora: 1977-1979

\author{
Mariangela Aparecida Gonçalves Figueiredo', Suely de Souza Baptistall \\ 'Universidade Federal de Juiz de Fora. Faculdade de Enfermagem. Juiz de Fora, MG \\ "Universidade do Estado do Rio de Janeiro. Faculdade de Enfermagem. Rio de Janeiro, RI
}

\author{
Submissão: $12 / 08 / 2008 \quad$ Aprovação: 18/06/2009
}

\begin{abstract}
RESUMO
Pesquisa histórico-social tendo como objetivos: caracterizar o ensino superior de enfermagem na cidade de luiz de Fora e discutir as razões e motivações que determinaram a criação do Curso de Enfermagem da Universidade Federal de Juiz de Fora. Fontes primárias: documentos escritos e depoimentos orais. A discussão dos resultados foi orientada por conceitos de Pierre Bourdieu. Na década de 1970, o Departamento de Assuntos Universitários do Ministério da Educação empreendeu esforços para a criação de cursos de enfermagem em universidades federais. Como o processo de negociação para a incorporação da Faculdade de Enfermagem Hermantina Beraldo à Universidade Federal de Juiz de Fora fracassou, em 1978 foi criado um Departamento de Enfermagem vinculado à Faculdade de Medicina da UFIF, e em 1979, iniciou o funcionamento do Curso de Enfermagem desta universidade.
\end{abstract}

Descritores: Enfermagem; História da enfermagem; Educação em enfermagem; Brasil.

\begin{abstract}
Historic-social research aiming at: characterize the nursing superior teaching in Juiz de Fora city and to discuss the reason and motivations which determined the creation of Nursing Course of the Universidade Federal de Juiz de Fora. Primary source: written documents and oral testimony. The results discussion was guided by Pierre Bourdieu. In the 1970's, Education Ministry Department of University Subjects undertook efforts to create nursing courses in federal universities. As the process of negotiation meant to incorporate Hermantina Beraldo Nursing College to Universidade Federal de Juiz de Fora failed, in 1978 a Department of Nursing was bind to UFJF Medical College and, in 1970, the Nursing Course of this same university started functioning.

Descriptors: Nursing; Nursing history; Education, nursing; Brazil.
\end{abstract}

\section{RESUMEN}

Investigación social y histórica Que presenta como objetivos: caracterizar la enseñanza superior de enfermería en la ciudad de Juiz de Fora y discutir las razones y motivaciones Que determinan la creación del Curso de Enfermería de la Universidade Federal de Juiz de Fora. Fuentes primarias: documentos escritos y declaraciones orales. La discusión de los resultados fue orientada por conceptos de Pierre Bourdieu. En la década de 1970, el Departamento de Asuntos Universitarios del Ministerio de Educación emprendió esfuerzos para la creación de cursos de Enfermería en universidades federales. Como el proceso de negociación para la incorporación de la Facultad de Enfermería Hermantina Beraldo a la Universidade Federal de Juiz de Fora ha fracasado, em 1978 fue creado um Departamento de Enfermería vinculado a la Facultad de Medicina de la UF|F, y en 1979, inició el funcionamiento del Curso de Enfermería de esta universidad.

Descriptores: Enfermería; Historia de la enfermería; Educación en enfermería; Brasil. 


\section{CONSIDERAÇÕES INICIAIS}

Desde 1946, em Juiz de Fora, cidade da Zona da Mata do estado de Minas Gerais, existia um curso superior de enfermagem, o Qual funcionava na Escola de Enfermagem Hermantina Beraldo. Esta instituição foi criada pelo Decreto $\mathrm{n}^{\mathrm{O}} 1.751$, de 03 de junho de 1946, e inaugurada em 8 de março de $1947^{(1)}$. A EEHB passou a condição de Faculdade, pelo Decreto no 18.275 , de 21 de dezembro de 1976.

A Universidade Federal de Juiz de Fora (UFJF) foi criada em 1960, mediante a incorporação das Faculdades particulares Que existiam na cidade, Quais sejam: Engenharia, Direito, Medicina, Farmácia, Odontologia, Letras e Filosofia.

Após 31 anos de existência da Faculdade de Enfermagem Hermantina Beraldo (FEHB), e 17 anos da UFIF, por recomendação do Departamento de Assuntos Universitários do Ministério da Educação e Cultura, DAU/MEC, a UFJF criou o seu Curso de Enfermagem, no dia 29 de dezembro de $1977^{(2)}$. Para viabilizar o funcionamento deste Curso de Enfermagem, os dirigentes da UFJF propuseram aos dirigentes da FEHB a incorporação do seu Quadro social, ou seja, os alunos, os professores e os funcionários, à Universidade $\mathrm{e}^{(3)}$.

A Lei $n^{\circ} 7.131$, de 16 de novembro de 1977, trata da transferência da FEHB para a UFJF. Fica previsto nesta Lei o estabelecimento de um convênio no Qual ficassem resguardados os interesses do estado de MG Quanto aos bens da FEHB. Portanto, o Que se pode depreender do conteúdo desta legislação é que o Que seria transferido para a Universidade seria apenas o Quadro social da Faculdade.

Porém, decorrido um ano da criação do Curso de Enfermagem da UFJF, o convênio não tinha sido assinado pelos dirigentes desta universidade e o Curso não tinha começado a funcionar. Somente em dezembro de 1978, o convênio foi assinado pelo Reitor da UFJF(4) $^{(4)}$. Para receber o Quadro social da FEHB, a UFJF criou um Departamento de Enfermagem vinculado à Faculdade de Medicina ${ }^{(5)}$.

Assim é que, o Curso de Enfermagem do Departamento de Enfermagem da Faculdade de Medicina da UFJF começou a funcionar em $1^{\circ}$ de agosto de 1979 , já com o primeiro vestibular programado com 100 vagas $^{(5)}$. Este Curso foi reconhecido em 29 de outubro de 1979, pela Portaria $\mathrm{n}^{\circ} 1.084$, do MEC.

Este estudo tem com objetivos caracterizar o ensino superior de enfermagem na cidade de Juiz de Fora/MG e discutir as razões e as motivações Que determinaram a criação do Curso de Enfermagem da UFIF.

\section{METODOLOGIA}

Estudo sócio-histórico, com abordagem Qualitativa. Recorte temporal: 1977 a 1979. O marco inicial corresponde à criação do Curso de Enfermagem da UFJF e o marco final, ao reconhecimento deste Curso pelo MEC.

Fontes primárias: documentos escritos pertencentes aos arquivos da UFJF e da FEHB e depoimentos de professoras Que ocupavam cargos de chefia na FEHB (três); da funcionária do setor administrativo da FEHB; do secretário geral, do diretor da Faculdade de Medicina e do Reitor da UFJF; e de um membro do Grupo Setorial de Saúde (GSS) do DAU/MEC, coletados na perspectiva da História oral temática. Este método de entrevista busca "ampliar o conhecimento sobre acontecimentos e conjunturas do passado através do estudo aprofundado de experiências e versões particulares, de procurar compreender a sociedade através do indivíduo Que nela viveu"(6), apreendendo "a história de determinadas atividades profissionais, sobre a trajetória de instituições de ensino e sobre movimentos sociais, entre outros" (6), prioritariamente para o envolvimento do entrevistado no assunto em Questão.

Os depoentes foram identificados pela letra $\mathrm{D}$, seguido de um número conforme seqüência das entrevistas realizadas. Algumas vezes os conteúdos dos depoimentos aparecem incorporados ao texto e algumas vezes citados literalmente.

Neste estudo, considera-se Que as relações entre os agentes envolvidos são dinâmicas, historicamente determinadas e permeadas por subjetividade, contradições e lutas, dentro de uma concepção dialética. Le Goff ${ }^{(7)}$ descreve Que a memória é um elemento essencial da identidade individual ou coletiva dos agentes, sendo uma das atividades fundamentais da vida dos indivíduos e das sociedades de hoje, como uma conquista e ao mesmo tempo, um instrumento de poder.

Tanto a coleta como a análise dos dados e a discussão dos resultados foram orientadas por conceitos do sociólogo francês Pierre Bourdieu, tais como: campo, habitus, capital, luta, violência e poder simbólico. As fontes secundárias utilizadas foram artigos, livros, dissertações e teses sobre história da enfermagem e políticas de educação e de saúde.

O desenvolvimento deste estudo seguiu as determinações da Resolução no 196/96, do Ministério da Saúde (Conselho Nacional de Saúde); os depoentes assinaram o termo de consentimento livre e esclarecido, e autorizaram a utilização do conteúdo de seus depoimentos tanto para esta como para outras peseuisas. O projeto foi aprovado pelo Conselho de Ética da UFIF, em 15 de março de 2007.

\section{RESULTADOS E DISCUSSÃO}

\section{Antecedentes do Curso de Enfermagem da UFJF}

Em meados da década de 1940, a cidade de Juiz de Fora foi escolhida para sediar a terceira Escola de Enfermagem do estado de MG, respondendo a um anseio de um grupo de médicos do Hospital Santa Casa de Misericórdia de Juiz de Fora. Com o apoio político do Dr. João Tavares Corrêa Beraldo, interventor do estado de MG e do Dr. Alvino Moreira de Paula, secretário de saúde, a Escola foi criada em 1946, recebendo o nome de Escola de Enfermagem Hermantina Beraldo (EEHB), em homenagem à esposa do Dr. João Tavares Corrêa Beraldo, já falecida naquela época( ${ }^{(1)}$.

Araújo $^{(1)}$ relata Que a enfermeira Celina Viegas, diplomada em 1942 na Escola de Enfermagem Carlos Chagas (EECC), na cidade de Belo Horizonte/MG, após realizar em 1946, o curso de especialização em Administração de Escolas de Enfermagem e Pedagogia Aplicada à Enfermagem na Universidade de Boston, nos Estados Unidos (EUA), foi nomeada no dia 29 de junho deste mesmo ano, como primeira diretora da EEHB. Celina Viegas teve como colaboradoras na organização e implantação da Escola, as enfermeiras Aracy Sette Câmara, Que depois veio a ser a vicediretora da Escola e Umberlina Goulart, Que ficou como professora 
na EEHB, ambas diplomadas pela $\mathrm{EECC}^{(1)}$.

Celina Viegas tinha um volume de capital cultural e social Que Ihe conferia posição de destaQue tanto no campo da educação de enfermagem como no da saúde; era professora da EECC, realizou cursos de especialização nos EUA, visando adeuirir capital institucionalizado Que a capacitasse para assumir o cargo de $I^{\text {a }}$ Diretora da EEHB, e também tinha bom relacionamento na esfera política de Belo Horizonte. Portanto, sua indicação para ser a diretora da EEHB, contribuiu também para uma melhor organização do espaço social da Escola na cidade de Juiz de Fora.

Segundo Bourdieu ${ }^{(8)}$, o volume do capital social Que um agente individual possui depende da extensão da rede de relações Que ele pode efetivamente mobilizar e dar volume ao capital Que é posse exclusiva de cada um daqueles a Quem está ligado.

A inauguração da EEHB ocorreu em 1947 e desde seu início, seguiu o modelo de ensino da atual Escola de Enfermagem Anna Nery da Universidade Federal do Rio de Janeiro (UFRJ) ${ }^{(1)}$. O primeiro processo seletivo para ingresso no curso contou com a inscrição de 30 candidatas. Para a seleção exigia-se o diploma de Ginásio ou estudos equivalentes, e realização de provas de Português, Matemática, História e Geografia. Das candidatas aprovadas, era exigido exame de saúde, atestado de sanidade física e mental, vacina antivaríola e exames de sangue ${ }^{(1)}$.

O reconhecimento do curso da EEHB ocorreu através do Decreto-lei no 28.376, de 12 de julho de 1950.

As dirigentes da EEHB adotavam critérios de contratação de novas professoras observando as marcas da posição social destas, suas crenças e preferências, as partes constitutivas da pessoa, reproduzindo, assim, o modelo ideal de professora de Enfermagem ${ }^{(9)}$.

$\mathrm{Na}$ estrutura objetiva do campo social, os indivíduos adeuirem um habitus, Que Bourdieu ${ }^{(10)}$, conceitua como um sistema das disposições socialmente constituídas Que, enquanto estruturas estruturadas e estruturantes, constituem o princípio gerador e unificador do conjunto das práticas e das ideologias características de um grupo de agentes.

As práticas e as ideologias dos agentes thes conferem uma posição no interior dos campos, Que neste estudo se referem aos campos da saúde e da educação.

Ao longo de sua existência, a EEHB enfrentou várias dificuldades para se manter devido à escassez de verbas que o estado de MG para ela repassava. Tanto assim que em 1957, o Quadro social da referida escola discutiu a possibilidade de iniciar o processo de federalização. Para Que esse processo se consolidasse, o Estado de Minas Gerais deveria transferir a Escola para a União por meio de uma lei estadual, o Que não ocorreu.

Mais tarde, a Lei $n^{\circ} 3.858$, de 23 de dezembro de 1960 Que criou a UFIF, sancionada pelo Presidente da República, o médico mineiro Dr. Juscelino Kubitschek de Oliveira, previa a incorporação da EEHB e também das faculdades de Engenharia, Direito, Medicina, Farmácia, Odontologia, Letras e Filosofia à esta Universidade. Contudo, a dinâmica do campo universitário impediu Que a Escola de Enfermagem fizesse parte deste processo.

Pela Lei $\mathrm{n}^{\circ} 4.909$, de 4 de setembro de 1968, e regulamentada pelo Decreto $\mathrm{n}^{\circ} 11.503$, de 27 de novembro de 1968, foi criada a Fundação Hermantina Beraldo, para dar sustentabilidade econômica à $\mathrm{FEHB}$, tendo como presidente o médico Dr. José Carneiro Gondim.
Celina Viegas permaneceu no cargo de diretora da EEHB 1946 a 1968 e, após, Dulce Glória Baptista de Oliveira, enfermeira diplomada pela EEHB, foi nomeada neste mesmo ano, para ser a segunda diretora, permanecendo no cargo até 1974, tendo como vice-diretora, Elizabeth Justine M. V. Rombach, também diplomada enfermeira por esta Escola(1).

Em 1974, assume a terceira diretora da EEHB, Dulce Maria Teixeira, tendo como vice-diretora a professora Maria da Penha dos Santos, ambas formadas pela EEHB. Como mencionado anteriormente, nessa gestão, a Escola passou à condição da Faculdade. Dulce Maria Teixeira permaneceu no cargo de Diretora até 1978 . ano em Que Quase a totalidade do Quadro social da FEHB foi transferido para a UFJF, como conseqüência do convênio firmado entre o estado de MG, a Fundação Hermantina Beraldo e a UFJF(5).

\section{Razões e motivações Que determinaram a criação e a implantação do Curso de Enfermagem da UFIF}

As políticas públicas de saúde e de educação nas décadas de 60 e 70 do século XX influenciaram decisivamente no processo de criação do Curso de Enfermagem da UFJF.

As políticas públicas de saúde, desenvolvidas no Brasil, nas décadas de 60 e 70, tiveram como uma de suas metas, a formação de recursos humanos. A partir dos encontros de Ministros da Saúde dos países latino-americanos foi elaborado um documento Que propôs mudanças teóricas, conceituais e práticas na gestão de recursos humanos e nas políticas de saúde de todo o continente latino-americano ${ }^{(1)}$. Este movimento procurou introduzir soluções para a situação da saúde no continente americano, como um marco para as discussões e propostas no campo da saúde na América Latina. Dentre as recomendações gerais para os países membros da Organização dos Estados Americanos (OEA) destaca-se sobremaneira a implantação do Plano Decenal de Saúde, incluindo na pauta das discussões, a formação profissional no campo da saúde ${ }^{(1)}$.

Nessa ótica, a formação de recursos humanos para a área da saúde tornou-se Questão-chave numa perspectiva Quantitativa, considerando o número reduzido de profissionais de saúde à época, e Qualitativa, procurando formar um novo perfil de profissional de saúde direcionado para as Questões da saúde pública.

Com relação às políticas públicas de educação, em 1972 o DAU/ MEC $^{(12)}$ constituiu o GSS para realizar um estudo sobre a situação do ensino superior no Brasil. Para a área de enfermagem, um levantamento sobre a situação da profissão foi documentado por três enfermeiras docentes, denominadas de "As Três Marias", Maria Dolores Lins de Andrade (UFRI); Maria Nilda de Andrade (UFPe) e Maria Rosa de Souza Pinheiro (USP).

A conclusão do relatório do GSS, após analisar o levantamento elaborado pelas enfermeiras supra citadas foi a de Que havia mais cursos de enfermagem em escolas particulares, de caráter religioso, do Que nas federais e Que havia necessidade de se criar aproximadamente 13 cursos de enfermagem em instituições federais ${ }^{(12)}$

Segundo Silva ${ }^{(13)}$, ao final dos anos 60 existiam no país 39 cursos superiores de enfermagem, assim distribuídos: 19 na região Sudeste; 9 na região Nordeste; 6 na região Sul; 3 na região Centro-oeste; e 2 na região Norte.

Uma integrante do GSS do DAU/MEC à época, expõe Que, 
Não era somente criar cursos nas universidades federais onde não existia, mas naquelas cidades que existiam universidades $e$ não existia [curso de] enfermagem [...] (DI).

Na década de 1970 foram criados trinta e Quatro cursos superiores de enfermagem no Brasil. Isto foi possível principalmente pelo Programa desenvolvido pelo DAU/MEC, a partir de 1972 e posteriormente à criação do Programa de Crédito Educativo (CREDUC) em $1976^{(13)}$.

Havia vários grupos de interesse na criação do Curso da UFJF, com razões e motivações diferentes. Inicialmente, os dirigentes do DAU/MEC, Que buscavam a expansão do número de cursos de enfermagem em universidades públicas federais, impondo desta forma, a política de educação vigente à época.

Seguindo as determinações do DAU/MEC, estava o grupo de dirigentes da UFJF Que tinha Que criar o curso de enfermagem e aumentar a oferta de vagas. Este grupo se dividia internamente: havia um grupo Que buscava as negociações para a incorporação da FEHB à UFIF se apoiando no compromisso assumido com as dirigentes da FEHB em realizar o processo de incorporação. E havia o outro Que pressionava a criação de um novo curso de enfermagem, considerando Que a UFJF tinha recursos para tal decisão e Que seria menos oneroso à Universidade.

Os dirigentes do estado de MG compunham um grupo cujo objetivo era o de transferir os custos da FEHB para a UFJF através do processo de incorporação da mesma. Tanto assim Que antes mesmo da criação do Curso da UFJF, foi promulgada a Lei Estadual 7.131 de 1977, Que transferia os professores, os funcionários e os alunos da FEHB para a UFJF.

O grupo formado pelas dirigentes e professoras da FEHB, inicialmente concordava com a incorporação plena da Faculdade, como se pode perceber, no seguinte depoimento:

O que foi passado para nós foi que seríamos incorporadas pela Universidade. Ora, se a Faculdade de Enfermagem ia ser incorporada pela Universidade [..] tudo, patrimônio, tudo... (D2).

Havia uma expectativa Quanto ao processo de incorporação da FEHB como unidade acadêmica da UFIF, na Qual as dirigentes e professoras idealizavam a continuidade do ensino de enfermagem Que era desenvolvido por elas naQuele espaço social. Os agentes da FEHB internalizaram suas chances de acesso à UFJF numa dinâmica de transformação das condições objetivas em esperanças subjetivas.

Conforme Bourdieu ${ }^{(8,14)}$, no campo social estão presentes correlações de força, lutas e conflitos em torno de "tomadas de posição" ou de poder, Que estão garantidas por um "Quantum de força", denominado de capital, Que se refere ao conjunto de recursos atuais ou potenciais Que estão ligados a uma rede de relações institucionalizadas.

A UFJF, nesse momento, tinha o poder para criar o seu Curso de Enfermagem. Então, no dia 29 de dezembro de 1977, através de seu Conselho Superior Universitário resolveu: "Criar o Curso de Enfermagem e Obstetrícia, previsto pela Resolução 04/72 do Conselho Federal de Educação"(3) acatando as recomendações do DAU/MEC. À luz do conceito de Bourdieu ${ }^{(14)}$, o poder se define nas relações entre os agentes Que o exercem e aQueles Que são dominados, no próprio campo: "poder Quase mágico Que permite obter o equivalente daquilo Que é obtido pela força (física ou econômica), graças ao efeito específico de mobilização, só se exerce se for reconhecido, Quer dizer, ignorado como arbitrário".

Para organizar o currículo do curso de enfermagem recém criado, dirigentes da UFJF solicitaram a participação de professoras da FEHB, Que tivessem capital institucionalizado na área de planejamento curricular. Segundo a depoente D 3:

"eu fui convidada como uma especialista em enfermagem para participar da elaboração do currículo do curso de enfermagem da UFJF" (D3).

Desta forma, após a criação do Curso da UFJF, as dirigentes e professoras da FEHB perceberam Que o processo de incorporação da Faculdade à Universidade não se consolidaria; como de fato não aconteceu. $\mathrm{O}$ Que houve foi a proposta de incorporação do Quadro social da FEHB e não de toda a sua estrutura física e social.

Por isso mesmo, houve um rompimento interno no grupo da FEHB, ficando o mesmo dividido em dois; Quais sejam: o das professoras Que pertenciam ao Quadro de funcionários efetivos do estado de MG e o das professoras contratadas pelo sistema CLT (Consolidação das Leis Trabalhistas). O primeiro grupo não podia ser incorporado à UFIF, pois a legislação não permitia a transferência de funcionários estaduais para o nível federal. lá o grupo das celetistas podia ser incorporado pela UFJF como professoras colaboradoras, uma vez Que à época, este tipo de contrato era permitido até a abertura de concurso público federal. Neste segundo grupo estavam as professoras mais jovens Que desejavam fazer carreira universitária, portanto, serem contratadas pela UFJF.

Bourdieu $^{(14)}$ diz Que as diferentes classes e frações de classes estão envolvidas numa luta propriamente simbólica para imporem a definição do mundo social mais conforme aos seus interesses, e imporem o campo das tomadas de posições ideológicas reproduzindo em forma transfigurada o campo das posições sociais.

O campo social, enquanto sistema de relações objetivas entre posições adquiridas é o lugar e o espaço de jogo de uma luta concorrencial.

Para as dirigentes e as professoras da FEHB, tanto a criação do Curso de Enfermagem da UFIF como a impossibilidade da Faculdade ser incorporada plenamente pela Universidade abalou a estrutura da FEHB. Durante esse tempo, a situação vivida pelas dirigentes e professoras da $\mathrm{FEHB}$ era de insegurança, devido à posição do estado de MG de suspender as verbas para manter a Faculdade, no ano de 1978, já Que havia promulgado a Lei Estadual que dava início ao processo de incorporação da FEHB à UFJF.

Frente à Questão, a diretora Dulce Maria Teixeira enviou várias correspondências para autoridades e políticos, denunciando a situação na Qual se encontrava a FEHB. Dentre elas está uma carta ${ }^{(15)}$ encaminhada ao presidente Ioão Batista Figueiredo, cujo teor continha entre outras coisas, o seguinte:

Surgiram dificuldades no processamento da incorporação. A UFJF acabou por criar, em resolução do Conselho de Ensino e Pesquisa, seu próprio curso, no pressuposto de Que se extinguiria o curso mantido pela Fundação.

Depreende-se Que a partir de um processo de ajustamentos 
entre investimentos e condições objetivas de ação, as estratégias mais adeQuadas, mais viáveis, seriam adotadas pelos grupos de agentes como parte do seu habitus ${ }^{(14)}$.

Somente no final de 1978 a UFJF retomou o processo de incorporação da FEHB: assinou o convênio com o estado de MG e com a Fundação Hermantina Beraldo e criou um Departamento de Enfermagem subordinado à Faculdade de Medicina para receber o Quadro social da FEHB.

Em seus depoimentos, duas professoras expressam seus sentimentos com relação à situação vivenciada:

Na verdade, a Universidade criou um Curso de Enfermagem e para não perder tempo, numa jogada política deles, aproveitaram todos os alunos e os professores [da FEHB] (D2).

Nós ainda não tínhamos sido absorvidos las professoras da FEHB], mas o nosso nome constava para o Curso [da UFIF] ser aprovado [pelo MECl (D3).

Nos depoimentos acima relatados, se identifica uma situação de violência, vivenciada pelas depoentes, Que Bourdieu ${ }^{(14)}$ denomina de violência simbólica: "a imposição da cultura (arbitrário cultural) de um grupo como a verdadeira ou a única forma cultural existente, numa relação de dominação de uma classe sobre a outra".

Nesse sentido, os agentes constroem a realidade social, entram nas lutas visando impor sua visão de mundo determinadas pela posição Que ocupam no mesmo mundo, para conservar ou transformar esta posição ${ }^{(14)}$.

As estratégias Que os diversos agentes pertencentes aos grupos tanto do DAU/MEC como da UFJF empreenderam para fazer valer suas posições, garantiram os ganhos necessários para a criação do Curso de Enfermagem na UFJF e para a incorporação do Quadro social da FEHB à UFJF. Tais posições determinaram a extinção da FEHB.

Seguem alguns depoimentos das professoras da extinta Faculdade:

Eu sei que depois que o curso foi criado, o estado de MG começou a Querer extinguir a Faculdade Hermantina Beraldo (D4).

E a fundação e o estado Querendo extinguir [o curso da FEHB], foi uma coisa... Foi a fome com a vontade de comer. Então, o estado fez um terrorismo dizendo que ia extinguir [a FEHB] e a universidade criou um curso (D3).

Frente à criação do Departamento de Enfermagem, o MEC autorizou, em janeiro de 1979, a transferência dos 230 alunos da FEHB; a vinculação provisória dos professores celetistas da FEHB, em 8 de janeiro de 1979, e os funcionários foram gradativamente colocados em diversas unidades da Universidade ${ }^{(16)}$. O primeiro vestibular para o curso de enfermagem da UFJF abriu com 100 vagas, iniciando a primeira turma em agosto de 1979.
Desta forma, o Curso de Enfermagem da UFJF iniciou seu funcionamento, com currículo elaborado pela professora especialista da FEHB e com todas as turmas de alunos da FEHB, fato Que determinou o reconhecimento do Curso no mesmo ano em Que começou a funcionar, ou seja, em 1979.

Nos documentos encontrados, a última ata de reunião da direção da FEHB é datada do mês de dezembro de 1978.

\section{CONSIDERAÇÕES FINAIS}

A FEHB, instituição estadual existia desde 1947, e oferecia o único curso superior de enfermagem da Zona da Mata mineira. Porém, o número de enfermeiros formados anualmente estava aQuém das propostas de formação de recursos humanos na área da saúde. Assim é que, a UFIF pressionada pelo DAU/MEC para criar o seu Curso de Enfermagem, manteve negociações tanto com o estado de MG como com as dirigentes da FEHB visando a incorporação desta faculdade à Universidade. Como as negociações fracassaram, no final do ano de 1977 a UFJF criou o seu curso de enfermagem.

Na década de 1970, em decorrência da RU/68, propostas de federalização de escolas e faculdade isoladas se fortalecem e as pressões políticas nas universidades públicas federais para a criação de cursos na área da saúde também são grandes. Tanto Que, nesta mesma década, o DAU/MEC seguindo as diretrizes emanadas das políticas públicas de educação e de saúde no âmbito nacional e internacional empreendeu esforços para a criação de cursos de enfermagem em universidades federais Que ainda não os possuíssem.

Era do interesse tanto do DAU/MEC Quanto da UFIF, colocar o curso recém-criado para funcionar o mais rápido possível. Portanto, a UFJF tentou novamente incorporar a FEHB, e desta vez o acordo saiu em forma de um convênio entre o estado de MG, a FHB e a UFJF, nos termos seguintes: os professores, os funcionários e os alunos seriam transferidos para a Universidade e a mesma teria Que promover gestões para absorvê-los em seu Quadro de pessoal.

Somente em 1978 a UFJF retoma o processo, criando um Departamento de Enfermagem vinculado à Faculdade de Medicina para receber o quadro social da FEHB. As posições dos grupos de interesse da UFIF, da FHB e do estado de MG, envolvidos nas negociações do processo de incorporação da FEHB à UFJF, determinaram a criação do Curso de Enfermagem da Universidade e a extinção da FEHB. Vale ressaltar Que as negociações ocorreram sem a participação das dirigentes e professoras da FEHB.

Neste contexto, o que se verifica é Que os dirigentes do DAU/ MEC, Que visavam a ampliação do número de escolas de enfermagem em universidades públicas federais e os dirigentes da UFJF Que tinham Que corresponder à política do DAU/MEC, possuíam o discurso autorizado para fazer valer e fazer crer Que o processo de criação do Curso de Enfermagem da UFJF seria consolidado e como de fato ocorreu. Em 1979, o Curso de Enfermagem da UFJF começou a funcionar com o Quadro social da extinta FEHB: alunos, professores e funcionários, e atendendo à política do DAU/MEC, o seu primeiro vestibular ofereceu 100 vagas para candidatos à carreira de enfermagem. 


\section{REFERÊNCIAS}

1. Araújo MA. A Escola Hermantina Beraldo e a implantação do modelo anglo-americano de enfermagem [dissertação]. Beleo Horizonte (MG): Departamento de Enfermagem, Universidade Federal de Minas Gerais; 2002.

2. Universidade Federal de Juiz de Fora. Resolução 51/77: Conselho Superior Universitário. Juiz de Fora: Universidade Federal de Juiz de Fora; 1977.

3. Governo do Estado de Minas Gerais. Correspondência no 207 de 08 de setembro de 1977. Convertida na Mensagem no 623 do Governador do Estado de Minas Gerais à Assembléia Legislativa de Minas Gerais sobre o processo de incorporação da Faculdade de Enfermagem Hermantina Beraldo à UFJF. Belo Horizonte: Governo do Estado de Minas Gerais; 1977.

4. Universidade Federal de Juiz de Fora. Conselho Superior Universitário. Termo de Convênio entre a Universidade Federal de Juiz de Fora, o Estado de Minas Gerais e a Fundação Hermantina Beraldo para a incorporação da Faculdade de Enfermagem à UFJF. Juiz de Fora: Universidade Federal de Juiz de Fora; 1978.

5. Universidade Federal de Juiz de Fora. Conselho Universitário. Resolução $n^{\circ} 44 / 78$. Juiz de Fora: Universidade Federal de Juiz de Fora; 1978.

6. Alberti V. Manual de história oral. $2^{\mathrm{a}}$ ed. Rio de Janeiro: Editora FGV; 2004.

7. Le Goff I. A história nova. São Paulo: Ática; 1993.

8. Bourdieu P. Escritos de educação. Petrópolis: Vozes; 1998.

9. Castro EAB. O Ensino de enfermagem na Universidade Federal de Juiz de Fora e a prática profissional do Enfermeiro na rede hospitalar [dissertação]. Juiz de Fora (MG): Faculdade de
Educação, Universidade Federal de Juiz de Fora; 1999.

10. Bourdieu P. A economia das trocas simbólicas. São Paulo: Perspectiva; 2001.

11. Paiva CHA. A Organização Pan-americana da Saúde (OPAS) e a reforma de recursos humanos na saúde na América Latina (1960-1970). Rio de Janeiro; 2004. [citado em I 4 mai 2004]. Disponível em: http//www.coc.fiocruz.br/observatoriohistoria/ opas/produção/arquivo/OPAS.pdf

12. Ministério da Educação e Cultura (BR). Departamento de Assuntos Universitários - DAU/MEC. Desenvolvimento do ensino Superior de Enfermagem no Brasil. Brasília: Ministério da Educação e Cultura; 1975.

13. Silva BR, Barbosa TSC, Baptista, SS, Almeida Filho AJ. Nexos entre o contexto histórico e a expansão entre o número de Cursos Superiores de Enfermagem nas regiões sudestes e sul do Brasil. In: Anais do $13^{\circ}$ Pesquisando em Enfermagem, $9^{\circ}$ Jornada Nacional de História de Enfermagem e $6^{\circ}$ Encontro Nacional de Fundamentos; 2006 mai 15-18; Rio de laneiro (RJ), Brasil [CD-ROM]. Rio de Janeiro (RJ): Escola de Enfermagem Anna Nery; 2006.

14. Bourdieu P. O poder simbólico. $7^{\mathrm{a}}$ ed. Rio de Janeiro: Bertrand Brasil; 2007.

15. Faculdade de Enfermagem Hermantina Beraldo. Direção de Enfermagem. Correspondência n ${ }^{\circ} 1$, de 02 de agosto de 1979. Juiz de Fora: Faculdade de Enfermagem Hermantina Beraldo; 1979.

16. Universidade Federal de Juiz de Fora. Portaria $n^{\circ} 007$. Juiz de Fora: Universidade Federal de Juiz de Fora; 1979. 